\title{
Los Determinantes del Nível de Precios en México: Un Enfoque Heterodoxo
}

\author{
Os determinantes do nível de preços no \\ México: um enfoque heterodoxo
}

LUIS MIGUEL GALINDO*

CARLOS GUERRERO**;***

RESUMO: O objetivo deste ensaio é analisar os efeitos do salário nominal mínimo, de um agregado monetário e da taxa de câmbio no nível de preços, usando o procedimento de Johansen para a co-integração. A evidência empírica indica a existência de uma relação de longo prazo entre essas variáveis e o nível de preço que pode ser interpretado como uma equação de preço. O modelo econométrico permite estabelecer os pesos específicos de cada uma dessas variáveis no nível de preços. Além disso, as evidências empíricas rejeitam a hipótese de fraca exogeneidade que confirma a existência de um feedback entre essas variáveis. Essas informações foram usadas para construir um modelo econométrico, a fim de simular e prever a dinâmica de preços no México, com propriedades estatísticas satisfatórias e estabilidade estrutural.

PALAVRAS-CHAVE: Inflação; salário mínimo; regime cambial.

ABSTRACT: The objective of this essay is to analyze the effects of the minimal nominal wage, a monetary aggregate and the exchange rate on the price level using the Johansen procedure for co-integration. The empirical evidence indicates the existence of a long-term relationship among these variables and the price level that can be interpreted as a price equation. The econometric model allows establishing the specific weights of each of these variables on the price level. Furthermore, the empirical evidence rejects the weak exogeneity hypothesis that confirms the existence of a feedback between these variables. This information was used to build an econometric model in order to simulate and forecast the dynamic of prices in Mexico with satisfactory statistical properties and structural stability.

KEYWORDS: Inflation; minimum wage; exchange rate regime.

JEL Classification: E31; F31.

\footnotetext{
* Profesor de la Facultad de Economía, UNAM, México. E-mail: gapaliza@servidor.unam.mx.

** Profesor de la Facultad de Economía, UNAM, México. E-mail: cguerrero@servidor.unam.mx..

*** Agradecemos los comentarios de Fidel Aroche, María Elena Cardero, lgnacio Perrotini, Eduardo Vega y Martín Puchet. La recopilación de la información estadística fue realizada por Kenia Ochoa y Eric Torres. Desde luego, la responsabilidad de los errores es exclusiva de los autores. Este proyecto fue financiado por PAPIIT: IN304197.
} 


\section{INTRODUCCIÓN}

La discusión sobre los determinantes de la inflación es ya vieja en América Latina. No obstante, continúan existiendo posiciones ciertamente diferenciadas. En efecto, existen posiciones divergentes que abarcan desde la visión de Harberger (1963), Vogel (1974) y Gómez-Oliver (1978) en la que el aumento de precios se explica fundamentalmente por las variables monetarias hasta aquellas como las de Noyola $^{1}$ (1987), Prebisch (1982) y Pinto (1968) en donde la inflación se origina en los desequilíbrios estructurales de carácter real y en los mecanismos de propagación. Además, existen propuestas intermedias como las de Díaz Alejandro (1965) que argumentan que los determinantes de la inflación incluyen elementos de costos y del mercado monetario.

La inexistencia de un acuerdo sobre la magnitud de los efectos que genera una devaluación de la moneda, una alza de los salarios nominales o un aumento de los agregados monetarios sobre el nivel de precios tiene consecuencias inmediatas tanto para las instituciones concentradas en la realización de pronósticos como para la toma de decisiones de política económica. Así, diversas instituciones del sector público, bancos o consultorias privadas estiman comúnmente los efectos de las variables arriba mencionadas sobre el nivel de precios con base en mediciones indirectas - tales como la composición entre bienes comerciables y no comerciables - o utilizan análisis parciales en los que se incluye una sola variable - como el tipo de cambio o los castos laborales unitarios -y, por tanto, se tiende a sobreestimar sus efectos sobre los precios.

Las simulacion es y proyecciones sobre la inflación en México se dificultan aún más porque esta variable parece comportarse como una senda aleatoria que incluye choques aleatorios pequenos pero permanentes, lo que se traduce en un persistente cambio en el valor de los coeficientes del modelo econométrico estimado y en dificultades para decidir sobre el orden de integración de las series empleadas (Webb, 1995 y Boschen y Talbot, 1991).

El estudio de la inflación con base a un modelo VAR y al procedimiento de Johansen (1988) permite construir un marco genera 1 para analizar conjuntamente la relevancia del salar io mínimo nominal, un agregado monetario y el tipo de cam bio respecto al nivel de precios. Además, este procedimiento econométrico permite determinar regias empíricas simples acerca de los efectos de cada una de estas va riables en lo s precios que pueden utilizarse para la realización de pronósticos y para propósitos de política económica.

El objetivo principal de este trabajo es la estimación de un modelo econométrico heterodoxo en el cual la estructura de la inflación depende de los castos laborales unitarios, de los efectos monetarios y de la inflación importada vía el tipo de cambio (De Brouwer y Ericsson, 1995). El trabajo se divide entres secciones. En la

\footnotetext{
${ }^{1}$ La inflación no es un fenómeno monetario; es el resultado de desequilíbrios de carácter real que se manifiestan en forma de aumentos del nivel general de precios” (Noyola, 1987, p. 67).
} 
primera establecemos el marco general del trabajo y la metodología econométrica utilizada. La segunda parte resume la evidencia empírica y, en la tercera sección, anotamos las conclusion es y algunos comentarias de política económica.

\section{MARCO TEÓRICO Y METODOLOGÍA ECONOMÉTRICA}

La teoría económica tiene diversas explicaciones sobre los determinantes del nivel de precios. Una clasificación de estas teorías considera las presiones originadas en los mercados laboral, de dinero, cambiario y de bienes ${ }^{2}$ (Martner, Titelman y Uthoff, 1995). Una especificación general puede formalizarse, asumiendo una función lineal homogénea (Mehra, 1991, Levy, 1984, Martner y Titelman, 1990, Webb, 1995, Juselius, 1992 y De Brouwer y Ericsson, 1995) como:

$$
P_{t}=U\left(C U_{t}, M_{t}, S_{t}, D E_{t}\right)
$$

donde $\mathrm{P}_{\mathrm{t}}$ representa el índice de precios, $\mathrm{CLU}_{\mathrm{t}}$ son los costos laborales unitarios ajustados por la productividad del trabajo y la tasa salarial o pago por trabajador, $\mathrm{M}_{\mathrm{t}}$ es el agregado monetario, $\mathrm{S}_{\mathrm{t}}$ es el tipo de cambio nominal y $\mathrm{DE}_{\mathrm{t}}$ es una variable de presión de demanda.

La determinación de los precios por los castos se desprende del modelo de competencia imperfecta (Carlin y Soskice, 1990) en donde los precios se determinan de acuerdo a un margen de ganancia ${ }^{3}$ sobre los castos laborales unitarios ya que los costos de los insumos sólo se transfieren:

$$
P_{t}^{\text {mex }}=\theta \mathrm{CLU}_{\mathrm{t}}
$$

Los costos laborales unitarios están definidos por:

$$
\mathrm{CLU}_{\mathrm{t}}=\left(\mathrm{W}_{\mathrm{t}} \mathrm{E}_{\mathrm{t}}\right) / \mathrm{Y}_{\mathrm{t}}
$$

donde $\mathrm{W}_{\mathrm{t}}$ es el salario nominal, $\mathrm{E}$, representa el nivel de empleo y $\mathrm{Y}_{\mathrm{t}}$ es el nivel de actividad económica. La tasa de crecimiento de los precios (L1P) se desprende de la ecuación (3):

$$
\Delta \mathrm{P}_{\mathrm{t}}=\Delta 1 \mathrm{CLU}_{\mathrm{t}}=\Delta \mathrm{W}_{\mathrm{t}}+\Delta\left(\mathrm{E}_{\mathrm{t}} / \mathrm{Y}_{\mathrm{t}}\right)
$$

Considerando que la inversa de $\mathrm{L} 1(\mathrm{E} / \mathrm{Y})$, es una aproximación al crecimiento de la productividad del trabajo (TIL), entonces la ecuación (4) puede escribir se como:

$$
\Delta P_{t}=\mathrm{LIW}_{\mathrm{t}}-\mathrm{TIL}_{\mathrm{Lt}}
$$

Los efectos del nível de la actividad económica o de las presiones de demanda pueden incluirse considerando que inciden en la productividad del trabajo a través

\footnotetext{
${ }^{2}$ Por ley de Walras el mercado de bienes puede quedar determinado residualmente una vez conocidas las soluciones de los otros tres mercados.

${ }^{3} \mathrm{El}$ margen de ganancia se supone como relativamente constante o al menos predecible en el largo plazo. Bajo esta condición los precios se determinan por la teoría de los costos normales (Layard, Nickell y Jackman, 1996).
} 
de las ecuaciones (4) o (5) y, atendiendo a la curva de Phillips aumentada (Sachs y Larrain, 1993) que indica que la tasa de crecimiento del salario nominal responde al comportamiento del desempleo asociado al ritmo de actividad y a las expectativas de inflación:

$$
\Delta \mathrm{W},=\varphi \mathrm{U}_{\mathrm{t}}+\Delta \mathrm{P}_{\mathrm{t}+1}^{\mathrm{e}}
$$

donde $\mathrm{U}_{\mathrm{t}}$ es la tasa de desempleo con $\varphi<0$ y, el supraíndice $e$ representa la inflación esperada por los agentes económicos.

La evidencia empírica para México sugiere que existe una retroalimentacion positiva entre los precios y los salarios (Dávila, Izey Morales, 1984, Salas y Ize, 1984, Ross, 1984, Yacaman, 1984 y Arellano y González, 1993).

La explicación del comportamiento del nivel de precios basada en el mercado de dinero sostiene que existe una relación de equilibrio entre el nivel de precios y la masa monetaria. Esta se desprende de la ecuación cuantitativa del dinero:

$$
\mathrm{M}_{\mathrm{t}} \mathrm{V}_{\mathrm{t}}=\mathrm{P}_{\mathrm{t}} \mathrm{Y}_{\mathrm{t}}
$$

donde $\mathrm{V}_{\mathrm{t}}$ representa a la velocidad de circulación y $\mathrm{Y}_{\mathrm{t}}$ es el nivel de transacciones aproximado por el nivel de ingreso. Despejando el nivel de precios en la ecuación (7) se obtiene:

$$
\mathrm{P}_{\mathrm{t}}=\left(\mathrm{M}_{\mathrm{t}} \mathrm{V}_{\mathrm{t}}\right) / \mathrm{Y}_{\mathrm{t}}
$$

Esta ecuación expresa, bajo el supuesto de una velocidad de circulación estable y de un nivel de actividad real exógeno, que la masa monetaria determina el nivel de precios de equilibrio (Hallman, Porter y Small, 1991).

En México la evidencia empírica muestra que las presiones de demanda, aproximadas por variables reales o monetarias, son relevantes para explicar el comportamiento de los precios aunque la relaciónes compleja (Ize y Vera, 1984, Ruprah, 1982 y Aceituno y Ruprah, 1982). En particular, Davila, Ize y Morales (1984) y Yacaman (1984) argumentan que existe evidencia de que los agregados monetarios causan en el sentido de Granger a los precios, aunque el efecto sea estadísticamente débil y, de que también los precios son sensibles a las presiones de demanda. Por su parte, Ize y Vera (1984) y Salas y Ize (1984) argumentan que probablemente la relación de causalidad va del agregado monetario al producto y por esta vía a los precios. Galindo (1997) encuentra que los precios están cointegrados con el agregado monetario y el ingreso y que existe una relación positiva entre los precios y el agregado monetario e inversa entre los precios y el ingreso, lo que también es confirmado por Salas y Ize (1984) atendiendo a diversos rezagos en el comportamiento de las variables.

El nivel de precios puede determinarse también por un componente externo utilizando como marco de análisis los llamados modelos escandinavos (Corbo, 1985 y Lindbeck, 1979). La ecuación (9) expresa que el índice de precios interno es un promedio ponderado de los precios internos y externos (Corbo, 1985 y Sachs y Larrain, 1993).

$$
\mathrm{P}_{\mathrm{t}}^{\text {mex }}=\left[\phi_{1}\left(\mathrm{P}_{\mathrm{t}-1}{ }^{\operatorname{mex}}\left(1+\Delta \mathrm{P}_{\mathrm{t}}\right)\right)\right]+\left[\phi_{2}\left(\mathrm{P}_{\mathrm{t}-1}{ }^{\text {ex }}\left(1+\Delta \mathrm{P}_{\mathrm{t}}^{\mathrm{us}}\right)\right) \mathrm{S}_{\mathrm{t}}\right]
$$

donde $\phi_{1}$ y $\phi_{2}$ representan ponderaciones específicas y los supraíndices mex y us 
indican a México y el resto del mundo respectivamente. La ecuación (9) indica los efectos del tipo de cambio a través de los costos externos sobre la inflación interna. En el caso en que el tipo de cambio se ajuste pasivamente a la hipótesis de la paridad de poder adquisitivo, entonces la inflación es esencialmente un fenómeno interno (Ize y Vera, 1984).

Para México la evidencia empírica indica que los componentes externos son relevantes para explicar el comportamiento de la inflación interna (Ize y Vera, 1984, Yacaman, 1984, Ize y Salas, 1984 y Arellano y González, 1993). En particular, existe evidencia de que los movimientos en el tipo de cambio nominal están asociados con espirales inflacionarias (Salas y Ize, 1984) y causan en el sentido de Granger a la inflación (Davila, Iza y Morales, 1984). No obstante, la magnitud del efecto de la inflación externa ha variado en las últimas dos décadas (Yacaman, 1984 y Davila, Ize y Morales, 1984).

La ecuación ${ }^{4}(1)$ permite analizar los canales de transmisión hacia la inflación con base a un modelo de vectores auto regresivos ${ }^{5}$ en donde los precios al consumidor se determinan como un sobreprecio sobre los costos domésticos e importados con un ajuste del mercado monetario ${ }^{6}$ ligado al comportamiento de la demanda agregada y por ajustes dinámicos ${ }^{7}$ (De Brouwer y Ericsson, 1995):

$$
\begin{aligned}
& \mathrm{p}_{\mathrm{t}}=\alpha_{01}+\sum \beta_{\mathrm{i}} \mathrm{p}_{\mathrm{t}-\mathrm{i}-1}+\sum \delta_{\mathrm{i}} \mathrm{m}_{\mathrm{t}-\mathrm{i}}+\sum \phi_{\mathrm{i}} \mathrm{w}_{\mathrm{t}-\mathrm{i}}+\sum \lambda_{\mathrm{i}} \mathrm{s}_{\mathrm{t}-\mathrm{i}}+\mathrm{u}_{\mathrm{tt}} \\
& \mathrm{m}_{\mathrm{t}}=\alpha_{02}+\sum \beta_{\mathrm{i}} \mathrm{p}_{\mathrm{t}-\mathrm{i}}+\sum \delta_{\mathrm{i}} \mathrm{m}_{\mathrm{t}-\mathrm{i}-1}+\sum \phi_{\mathrm{i}} \mathrm{w}_{\mathrm{t}-\mathrm{i}}+\sum \lambda_{\mathrm{i}} \mathrm{s}_{\mathrm{t}-1}+\mathrm{u}_{2 \mathrm{t}} \\
& \mathrm{w}_{\mathrm{t}}=\alpha_{03}+\sum \beta_{\mathrm{i}} \mathrm{p}_{\mathrm{t}-\mathrm{i}}+\sum \delta_{\mathrm{i}} \mathrm{m}_{\mathrm{t}-\mathrm{i}}+\sum \phi_{\mathrm{i}} \mathrm{w}_{\mathrm{t}-\mathrm{i}-\mathrm{1}}+\sum \lambda_{\mathrm{i}} \mathrm{s}_{\mathrm{t}-\mathrm{i}}+\mathrm{u}_{3 \mathrm{t}} \\
& \mathrm{s}_{\mathrm{t}}=\alpha_{04}+\sum \beta_{\mathrm{i}} \mathrm{p}_{\mathrm{t}-\mathrm{i}-1}+\sum \delta_{\mathrm{i}} \mathrm{m}_{\mathrm{t}-\mathrm{i}}+\sum \phi_{\mathrm{i}} \mathrm{w}_{\mathrm{t}-\mathrm{i}}+\sum \lambda_{\mathrm{i}} \mathrm{s}_{\mathrm{t}-1-1}+\mathrm{u}_{4 \mathrm{t}}
\end{aligned}
$$

las letras en minúsculas representan los logaritmos naturales de las series. Así, el VAR de la ecuación (9) puede representarse en su forma general como:

\footnotetext{
${ }^{4}$ Se considera que todos los efectos de la inflación externa están incluídos en los movimientos del tipo de cambio nominal y los autoregresivos de la inflación pueden entenderse como las variables que capturan los efectos inerciales.

${ }^{5}$ Puede decirse que este VAR estima lo que se conoce como la inflación dura, core inflation (Quah y Vahey, 1995).

${ }^{6}$ Es conveniente considerar que los coeficientes de esta variable pueden ser relativamente instables en la medida en que se observen modificaciones en los regímenes monetarios.

${ }^{7} \mathrm{La}$ inclusión de las presiones de demanda a través de un agregado monetario se debe a la evidencia empírica disponible y a consideraciones econométricas. En efecto, Webb (1995) y Hallman, Porter y Small (1991) para Estados Unidos, Hall y Milne (1994) para el Reino Unido y Galindo (1997) para México, encuentran que M2 es relevante para explicar el comportamiento del nivel de precios. Asimismo, existe evidencia para nuestro país de que el efecto del ingreso sobre los precios está asociado a la cantidad de dinero (Salas y Ize, 1984). Además, debe mencionarse que exis en consideraciones estadísticas relevantes para excluir e1 uso de variables que aproximen las presiones de demanda porque implica utilizar variables 1(0) como De Brouwer y Ericsson (1995) quienes emplean la diferencia entre el producto y su tendencia estimada o un estimado de la capacidad ociosa y que, por tanto, implicaría estimar una ecuación no balanceada que dificultaría el análisis de cointegración. Finalmente, Bruno (1979) y Sawyer, Aaronovitch y Samson (1982) encuentran en sus estimaciones econométricas que el efecto de las presiones de demanda sobre los precios es mínimo - para los casos de Inglaterra, Israel y E. U. - y nulo - para el caso de Suecia.
} 


$$
\mathrm{X}_{\mathrm{t}}=\Pi_{1} \mathrm{x}_{\mathrm{t}-1}+\ldots+\Pi_{\mathrm{k}} \mathrm{X}_{\mathrm{t} \_\mathrm{k}}+\Phi \mathrm{D}_{\mathrm{t}}+\mathrm{u}_{\mathrm{t}}
$$

donde $X_{t}$ representa un vector que contiene a todas las variables endógenas y $D_{t}$, es el vector que incluye a la constante y/o tendencia y a las variables estacionales dummy.

Reparametrizando el VAR de la ecuación (10) en su forma de corrección de errores (Johansen, 1992) se obtiene:

$$
\Delta \mathrm{X}_{\mathrm{t}}=\Gamma_{1} \Delta \mathrm{X}_{\mathrm{t}-1}+\ldots+\Gamma_{\mathrm{k}-1} \Delta \mathrm{X}_{\mathrm{t}-\mathrm{k}+1}+\Pi \mathrm{X}_{\mathrm{t}-\mathrm{k}}+\Phi \mathrm{D}_{\mathrm{t}}+\mathrm{u}_{\mathrm{t}}
$$

donde:

$$
\begin{aligned}
& \Pi_{1}=-\left(\mathrm{I}-\Pi_{1}-\ldots-\Pi_{\mathrm{k}}\right) \\
& \Gamma_{1}=-\left(\mathrm{I}-\Pi_{1}-\ldots-\Pi_{1}\right)
\end{aligned}
$$

En el caso en que Xt-k se componga de variables no estacionarias de orden 1(1) entonces LIX, contendrá exclusivamente variables estacionarias (1(0)). De este modo, todos los términos incluídos, con la excepción de $\mathrm{X}_{\mathrm{t}-\mathrm{k}}$ son variables estacionarias. El método de Johansen (1988) utiliza correlaciones canónicas para estimar las distintas combinaciones de $\mathrm{X}_{\mathrm{t}-\mathrm{k}}$ que producen correlaciones elevadas con los términos $1(0)$. Así, para que $\Pi \mathrm{X}_{\mathrm{t}-\mathrm{k}}$ contenga una combinación lineal de las variables que sea estaciognaria se requiere que exista un conjunto de vectores de cointegración o que esta matriz contenga solo ceros como coeficientes que se denomina como la solución trivial. La matriz $\Pi^{8}$ de coeficientes de $\mathrm{X}_{\mathrm{t}}$ puede descomponerse entonces en dos matrices:

$$
\Pi=\alpha \beta \text { ' }
$$

En el caso de la existencia de una matriz tal que:

$$
\beta^{\prime} \mathrm{X}_{\mathrm{t}-\mathrm{k}} \rightarrow 1(0)
$$

Las columnas de $\beta$ ' forman un vector de parámetros de cointegración de $\mathrm{X}_{\mathrm{t}-\mathrm{k}}$. $\mathrm{y}$, por tanto, de $\mathrm{X}_{\mathrm{t}}$. La solución trivial se descarta y consecuentemente, se espera al menos k-1 vectores de cointegración. En el caso en que las series no estén cointegradas entonces $\beta$ ' sería una matriz nula.

La matriz a se basa en la estimación de la factorización de los coeficientes incluidos en este vector. Así, considerando el caso que incluye sólo la primera columna de $\alpha \beta$ ', entonces la ecuación (13) puede escribirse como:

$$
\Delta \mathrm{X}_{\mathrm{t}}=\Gamma_{1} \Delta \mathrm{X}_{\mathrm{t}-1}+\ldots \Gamma_{\mathrm{k}-1} \Delta \mathrm{X}_{\mathrm{t}-\mathrm{k}+1}-\alpha \mathrm{Z}_{\mathrm{t}-\mathrm{k}} \cdot+\Phi \mathrm{D}_{\mathrm{t}}+\mathrm{u}_{\mathrm{t}}
$$

donde $Z_{t}=\beta X_{t}$, En este sentido, los coeficientes de a pueden interpretarse como los coeficientes del término de corrección de errares de la ecuación (15) (Engle y Granger, 1987).

La hipótesis de cointegración considera el caso donde la matriz П contiene una combinación lineal con su espacio definido en $\mathrm{I}(0)$ :

$$
\text { Ho: }=\Pi=\alpha \beta \text { ' }
$$

\footnotetext{
${ }^{8}$ La matriz $\Pi$ es de orden kxn donde k es el número de variables y $\mathrm{r}$ el número de datas. Se denota como $\mathrm{r}$ al número de vectores de cointegración.
} 
Esta hipótesis se resuelve bajo tres casos (Cuthbertson, Hall y Taylor, 1992):

1) La matriz $n$ tiene rango completo. Ello implica que $X_{t}$ es estacionaria.

2) La matriz TI tiene rango cero y por tanto las series no cointegran.

3) EI rango de la matriz TT es menor que $\mathrm{k}$ - el numero de variables incluídas - y por tanto, existe una combinación lineal de las variables no estacionarias que es 1(0) indicando que las series están cointegradas. De este modo, el concepto de cointegración entre las variables implica la existencia de al menos k-1 vectores de cointegración. Las pruebas de cointegración por el procedimiento de Johansen pueden desprenderse de la ecuación (14) al despejarse como:

$$
\Delta \mathrm{X}_{\mathrm{t}}+\alpha \beta^{\prime} \mathrm{X}_{\mathrm{t}-\mathrm{k}}=\Gamma_{1} \Delta \mathrm{X}_{\mathrm{t}-1}+\ldots+\Gamma_{\mathrm{t}-\mathrm{k}-1} \Delta \mathrm{X}_{\mathrm{t}-1}+\Phi \Delta \mathrm{t}+\mathrm{u}_{\mathrm{t}}
$$

De este modo, cancelando el efecto generado por los términos en primeras diferencias en el lado derecho de la ecuación (17) quedan solamente los residuales. Esto puede realizarse al remplazar $\Delta \mathrm{X}_{\mathrm{t}-1} \mathrm{y} \mathrm{X}_{\mathrm{t}-\mathrm{k}}$ con los residuales si se estiman estas variables con respecto a $\Delta \mathrm{X}_{\mathrm{t}-1}, \ldots, \Delta \mathrm{X}_{\mathrm{t}-\mathrm{k}+1}$ en forma individual (Cuthbertson, Hally Taylor, 1992). De este modo, la ecuación (17) puede escribirse como:

$$
\mathbf{R}_{0 \mathrm{t}}+\alpha \beta^{\prime} \mathrm{R}_{\mathrm{kt}}=\mathrm{u}_{\mathrm{t}}
$$

donde $\mathrm{R}_{0 \mathrm{t}}$, es un vector de residuales de la regresión de $\Delta \mathrm{X}_{\mathrm{t}}$ en $\Delta \mathrm{X}_{\mathrm{t}-1}, \ldots, \Delta \mathrm{X}_{\mathrm{t}-\mathrm{k}+\mathrm{I}} \mathrm{y}$ $\mathrm{R}_{\mathrm{kt}}$ es el vector de residuales de la regresión de $\mathrm{X}_{\mathrm{t}-\mathrm{k}}$ con respecto a $\Delta \mathrm{X}_{\mathrm{t}-1}, \cdots, \Delta \mathrm{X}_{\mathrm{t}-\mathrm{k}+\mathrm{l}} \cdot{ }^{\circ}$

La función de máxima verosimilitud de esta ecuación puede expresarse como (Engle, 1983):

$$
\mathrm{L}(\alpha, \beta, \Omega)=|\Theta| \cdot \mathrm{N} / 2 \exp \left[-1 / 2 \Sigma^{\mathrm{N}}\left(\mathrm{R}_{0 \mathrm{t}}+\alpha \beta^{\prime} \mathrm{R}_{\mathrm{kt}}\right)^{\prime} \Omega^{-1}\left(\mathrm{R}_{0 \mathrm{t}}+\alpha \beta^{\prime} \mathrm{R}_{\mathrm{kt}}\right)\right]
$$

donde $\Omega$ representa la matriz de covarianzas y $\Theta$ se obtiene de la regresión de $R_{0 t}$ en $\beta^{\prime} R_{k t}$ y $N$ es el número de observaciones. En este caso, $\alpha$ y $\Theta$ pueden expresarse en función de $\beta$ como:

$$
\begin{aligned}
& \alpha(\beta)=-S_{0} \beta\left(\beta^{\prime} S_{k k} \beta\right)^{-1} \\
& \Omega(\beta)=S_{00}-S_{\text {ok }} \beta\left(\beta^{\prime} S_{k k} \beta\right) \beta S_{k O} \\
& S=N^{-1} \Sigma^{T} R_{i t} R^{\prime} \text {, donde i,j=0,k }
\end{aligned}
$$

Sustituyendo (20.a) y (20.b) en (19) se obtiene la función de máxima-verosimilitud concentrada que puede expresarse como:

$$
\mathrm{L}_{2}(\beta)|\Omega \beta|^{-\mathrm{N} / 2}=\left|\mathrm{S}_{00}-\mathrm{S}_{0 \mathrm{k}} \beta\left(\beta^{\prime} \mathrm{S}_{\mathrm{kk}} \beta^{\prime} \mathrm{S}_{\mathrm{k} 0}\right)\right|
$$

Por tanto, la función de máxima verosimilitud permite obtener los vectores de cointegración minimizando $\beta$. Esta matriz de betas se obtiene con base en el procedimiento de raíces y vectores característicos y a una prueba de razón de máximaverosimilitud. Consecuentemente, los estadísticos utilizados son las pruebas de la raíz característica máxima y de la traza (Johansen, 1992):

$$
\begin{aligned}
& \lambda_{\max }=-\mathrm{N} \ln (1-\lambda \\
& \mathrm{LR}(\mathrm{N}-\mathrm{r})=-2 \ln (\mathrm{Q})=-\mathrm{N} \Sigma^{\mathrm{N}} \ln (1-\lambda)
\end{aligned}
$$

Donde $\lambda$ representa la raíz característica y $Q$ la función de máxima-verosimilitud restringida dividida por la función de máxima verosimilitud sin restringir. Desafortunadamente la distribución de estas estadísticos no es como una chi cuadrada. Sin 
embargo, Johansen calculó su distribución y derivó los valores críticos con base al movimiento browniano (Cuthberson, Hall y Taylor, 1992).

Las pruebas de exogeneidad débil pueden definirse considerando al proceso generador de información (Spanos, 1986) como una función que transforma un conjunto de variables, condicionadas a un conjunto de información, en un modelo que determina las variables endógenas en función de las variables exógenas y margina a las variables no relevantes (Granger, 1990):

$$
\mathrm{F}\left(\mathrm{X}_{\mathrm{i}} / \mathrm{X}_{\mathrm{t}-1,}, \theta\right)=\mathrm{F}_{\mathrm{y} / \mathrm{z}}\left[\mathrm{Y}_{\mathrm{t}} / \mathrm{Z}_{\mathrm{t}}, \mathrm{X}_{\mathrm{t}-\mathrm{i}}, \mathrm{\Psi}_{1}\right]^{*} \mathrm{~F}_{\mathrm{z}}\left[\mathrm{Z}_{\mathrm{t}} / \mathrm{X}_{\mathrm{t}-1}, \Psi_{2}\right]
$$

donde $\mathrm{X}_{\mathrm{t}}=\left(\mathrm{Y}_{\mathrm{t}}, \mathrm{Z}_{\mathrm{t}}\right)$ representa la muestra total de los datos, $\mathrm{X}_{\mathrm{t}}$ es el total de las variables, $\mathrm{Y}_{\mathrm{t}}$ es el conjunto de las variables incluidas en el modelo, $\mathrm{Z}_{\mathrm{t}}$ son las variables marginadas del modelo, $\theta$ es el conjunto completo de parámetros de la distribución conjunta, $\Psi_{1}$ es el conjunto de parámetros del modelo condicional y, $\backslash \mathrm{j} / 2$ es el conjunto de parámetros del modelo marginal. De este modo, se define a las variables en $\mathrm{z}$, que cumplen la condición de exogeneidad débil si los parámetros de interés $\Psi_{1}$ pueden reparametrizarse de forma tal que $\Psi=\left(\Psi_{1}, \Psi_{2}\right)$; se cumpla que los parámetros de interés de 8 son una función de $\Psi_{1}$ y, la factorización presentada en la ecuación (23) permita operar adecuadamente un corte secuencial para diferenciar los parámetros $\Psi_{1}$ de $\Psi_{2}$ (Engle, Hendry y Richard, 1983). Así, los parámetros de $\Psi_{1}$ pueden modelarse solos sin incluir información sobre $\Psi_{2}$.

Consecuentemente, $\Psi_{1}$ y $\Psi_{2}$ varían de forma independiente y, por tanto, $\Psi_{2}$ no contienen información relevante para determinar los rangos de los valores de los coeficientes de interés (Ericsson y Irons, 1994). Debe considerarse que en el caso de variables no estacionarias 1(2), la reparametrizacion del VAR incluye dos diferencias e implica por tanto que las pruebas de exogeneidad débil pierdan fuerza (Johansen, 1992). Sin embargo, en esencia, el procedimiento continúa siendo el mismo (Johansen, 1992).

\section{EVIDENCIA EMPIRICA}

Las pruebas de raíces unitarias de Dickey Fuller y Phillips y Perron, sintetizadas en el Cuadro 1, indican que el índice de precios, el tipo de cambio nominal, el salario mínimo nominal y el agregado monetario $\left(\mathrm{m} 2_{\mathrm{t}}\right)$ son series de tiempo no estacionarias de orden 1(2). Es conveniente tomar con precaución los resultados de estas pruebas considerando que el número de datos utilizado en el trabajo es ciertamente limitado, ya que los valores críticos de las pruebas son normalmente derivados utilizando entre 100 y 150 repeticiones (Hatanaka, 1996). Si bien la informacion disponible es limitada dado el tamaño de la muestra, existen trabajos realizados sobre este tema con un número similar de claros (entre 53 y 74 observaciones) (Cuthbertson, Hall y Taylor, 1992, p. 23 y p. 28). 
Cuadro 1: Pruebas de raíces unitarias

\begin{tabular}{ccc}
\hline Variable & ADF(4) & PP(4) \\
\hline$p_{t}$ & 0.90 & 2.45 \\
$\Delta p_{t}$ & -1.54 & -1.40 \\
$\Delta^{2} p_{t}$ & $-3.11^{* *}$ & $-6.00^{* *}$ \\
$w_{t}$ & 0.19 & 1.10 \\
$\Delta w_{t}$ & -1.64 & $-3.76^{* *}$ \\
$\Delta^{2} w_{t}$ & $-3.35^{* *}$ & $-11.90^{* *}$ \\
$m_{t}$ & 0.71 & 3.70 \\
$\Delta m_{t}$ & -1.26 & $-4.49^{* *}$ \\
$\Delta^{2} m_{t}$ & $-3.03^{* *}$ & $-17.02^{* *}$ \\
$s_{t}$ & -0.17 & -0.42 \\
$\Delta s$ & -1.88 & -3.89 \\
$\Delta^{2} s_{t}$ & $-3.65^{* *}$ & $-14.98^{* *}$ \\
\hline
\end{tabular}

Notas:

ADF(4)=Pru ebas de D1c key Fuller Aumentada (1981) para corregir po,s b le s problemas de autoco rr e lación o heteroscedast,cidad

PP(4)=Pruebas de Phillips y Perron (1988).

Las pruebas de raíces unttarias no ,ncluyen constante o tendencia. Período · 1986(2)-1996(4).

Los resultados de las pruebas de raíces unitarias indican que el procedimiento de Johansen es adecuado para analizar estas series. Sin embargo, el orden de integración de las variables dificulta la interpretación de los resultados ya que pueden existir soluciones múltiples y combinaciones lineales de los vectores que pueden interpretarse como posibles soluciones (Wickens, 199 3) y, como se mencionó anteriormente, las pruebas de exogeneidad débil pierden fuerza.

El modelo de vectores autoregresivo (VAR) indica que es viable contruir un modelo con las propiedades estadísticas adecuadas. Esto es, los errores del VAR no muestran signos de autocorrelación o heteroscedasticidad y, los errores no rechazan la prueba de normalidad con las excepciones del agregado monetario y el tipo de cambio (cuadro 2 a del Apéndice). ${ }^{9}$ En el tipo de cambio esto se explica por la presencia de valores extremos asociados a sus movimientos bruscos.

Las pruebas de cointegración basadas en el procedimiento de Johansen, sintetizadas en el Cuadro 2, indican la presencia de al menos tres relaciones estables de largo plazo entre las variables estudiadas.

El mayor vector característico, asociado a la raíz característica máxima, puede interpretarse como una ecuación de precios determinada por los costas laborales unitarios, el agregado monetario y el tipo de cambio.

\footnotetext{
${ }^{9}$ Una definición de las pruebas econométricas utilizadas está en Doornick y Hendry (1997a) y (1997b).
} 
Cuadro 2: Pruebas de Johansen sobre cointegración

\begin{tabular}{ccccccc}
\hline Ho: rango $=p$ & - Tlog(1-Imu) & T-nm & $95 \%$ & -Tsumlog(.) & T-nm & $95 \%$ \\
\hline $\mathrm{P}==0$ & $47.07^{*}$ & $29.56^{*}$ & 27.1 & $76.83^{* *}$ & $48.29^{*}$ & 47.2 \\
$\mathrm{P}<=1$ & 20.27 & 12,73 & 21.0 & $29.76^{*}$ & 18.69 & 29.7 \\
$\mathrm{P}<=2$ & 5.68 & 3.56 & 14.1 & 9.49 & 5.96 & 15.4 \\
$\mathrm{P}<=3$ & $3.81^{*}$ & 2.39 & 3.8 & $3.81^{*}$ & 2.39 & 3.8 \\
\hline
\end{tabular}

Notas:

- Tlog(1-Imu)=prueba de la raíz característica máxima

- Tsumlog(.)= prueba de la traza

El modelo incluye constante sin restringir pero excluye a la tendencia.

VAR con cuatro rezagos. Período: 1986(2)-1996(4)

En efecto, la ecuación (23) indica que el nivel de precios tiene una relación positiva de largo plazo con el salario mínimo nominal, el agregado monetario y el tipo de cambio nominal. Destaca que el salario mínimo nominal es la variable con la mayor influencia sobre el nivel de precios como resultado probablemente, en primer lugar, de la estructura de costos o ponderación entre los costos salariales y externos de las empresas y, en segundo lugar, a que parte del efecto de los costos de la devaluación se transmite a los costos laborales unitarios en la medida en que también los salarios se ajustan ante una devaluación de la moneda. ${ }^{10}$ Esto último origina un aumento en la intensidad de la pugna distributiva que puede entenderse como un mecanismo de propagación (Fairlie, 1992).

$$
\mathrm{P}_{\mathrm{t}}=.52 \mathrm{w}_{\mathrm{t}}+.31 \mathrm{~s}_{\mathrm{t}}+.26 \mathrm{~m}_{\mathrm{t}}
$$

La evidencia empírica es favorable a las hipótesis de formación de precios de costos normales (Ross, 1981) o de sensibilidad de algunos precios clave a la inflación (Gil-Díaz y Ramos Tercero, 1988) aunado a un efecto de propagación o de validación de la inflación asociado al comportamiento del agregado monetario. En efecto, los estudios empíricos sobre la formación de precios en México parecen validar tanto la hipótesis de costos normales con diversas características sectoriales (Ross, 1981, Casar, et al, 1979 y Jimenez y Roces, 1979), como a la hipótesis que asocia los precios al comportamiento de la masa monetaria (Leiderman, Liviatan y Thorne, 1995).

Los resultados obtenidos tienen consecuencias relevantes desde el punto de vista de la política económica. En efecto, la existencia de una relación de largo plazo entre los niveles de esta variables indica que es posible utilizar al tipo de cambio, al salario mínimo nominal y al agregado monetario como anelas nominales del nivel de precios (Arellano y González, 1993 y Leiderman, Liviatan y Thorne, 1995). Esto es, el monto del salario mínimo nominal, el tipo de cambio y el agregado monetario generan un nivel de precios de equilíbrio consistente con estas

\footnotetext{
${ }^{10}$ Este mismo argumento parece válido para el agregado monetario. Esto es, una devaluación o un aumento de los costos laborales se transmite a la masa monetaria y por tanto, el freto individual parece pequeño aunque el efecto conjunto sea considerable.
} 
variables. Así, cuando los precios reales están por abajo de este nível de equilíbrio existirán presiones en la economía para una alza de precios y condiciones monetarias que lo validen. Por el contrario, cuando el nivel de precios de equilíbrio está por encima del nível de precios real, entonces existen condiciones para una deflación o al menos para que la evolución del índice de precios tienda a rezagarse.

Cuadro 3: Coeficientes $\beta$ del procedimiento de Johansen

\begin{tabular}{cccc}
\hline$P_{t}$ & $w_{t}$ & $s_{t}$ & $m_{t}$ \\
\hline 1.00 & -0.52 & -0.31 & -0.26 \\
-1.19 & 1.00 & 0.21 & 0.19 \\
-1.46 & 0.86 & 1.00 & -0.22 \\
-5.10 & 1.47 & 3.57 & 1.00 \\
\hline
\end{tabular}

Las pruebas de máxima-verosimilitud de restricción en los parámetros, sintetizadas en el Cuadro 4, indican que todas las variables incluidas son relevantes para explicar al nível de precios ya que se rechaza la hipótesis nula de que alguno de estos coeficientes sea cero. Consecuentemente, la exclusión del salario mínimo nominal, el agregado monetario o el tipo de cambio implica el uso de un modelo incorrectamente especificado que puede traducirse en estimadores sesgados y, por tanto, en una sobrevaluación del impacto de algunas de estas variables en los precios. El mayor impacto del salario mínimo nominal se confirma al observarse que la prueba de máxima-verosimilitud no rechaza la hipótesis nula de que el valor del coeficiente de $\mathrm{w}_{\mathrm{t}}$ es la suma de los coeficientes de $\mathrm{m}_{\mathrm{t}} \mathrm{y}_{\mathrm{t}}$. Este resultado difiere de Lizondo (1992) para quien las variables más relevantes son el agregado monetario y la inflación importada mientras que el efecto del salario sobre los precios es relativamente menor en México. Asimismo, la evidencia estadística rechaza que la suma de estos coeficientes sea igual a uno lo que es consistente con la evidencia encontrada por Corbo (1985) para Chile, Sahling (1977) para Estados Unidos y, De Brouwer y Ericsson (1995) para Australia. Esto puede interpretarse como consecuencia de los efectos que genera los cambias en el margen de ganancias de las empresas. Así, en el caso en que la suma de las elasticidades es menor a uno implica que el margen de ganancia aumentó en el período estudiado y viceversa.

Cuadro 4: Pruebas de máxima-verosimilitud sobre el valor de los parâmetros.

\begin{tabular}{ccc}
\hline$\beta_{1}=\beta_{2}=\beta_{3}=0$ & $\beta_{1}=\beta_{2}+\beta_{3}$ & $\beta_{1}+\beta_{2}+\beta_{3}=1$ \\
\hline$X^{2}(3)=40.01[.000]^{* *}$ & $X^{2}(1)=1.781[.181]$ & $X^{2}(2)=22.65[0.00]^{* *}$ \\
\hline
\end{tabular}

Nota: La especificación utilizada fue : $P_{1}=b_{1} w_{1}+b_{2} s_{1}+b_{3} m_{1}$.

La prueba de exogeneidad débil es rechazada por los datos $\left[\mathrm{X}^{2}(3)=\right.$ $34.76(.000) * *$ implicando que la formación de las variables estadísticas incluídas en el modelo no son independientes y por tanto, que existe información relevante en cada una de ellas para simular y predecir adecuadamente el nivel de precios (Ericsson e Irons, 1994). Este resultado es consistente con Mehra (1991) y Juselius (1992) para el caso de los países desarrollados, con Ruprah (1984) para México y, 
en términos generales, con Harberger (1963) y Díaz-Alejandro (1965) quienes también argumentan en favor de la simultaneidad entre las tasa de crecimienro de los precios, los salarios y un agregado monetario para Chile y Corbo (1985) quien argumenta sobre la retroalimentación entre los precios, los salarios y el tipo de cambio.

En resumen, los autores anteriormente mencionados argumenta la presencia de una retroalimentación entre los precios y los salarios y, entre los precios y el agregado monetario. Este resu lt ado común sugiere la conveniencia de tomar con precaución al modelo econométrico final ya que el cambio brusco en alguna de las variables puede incidir sobre el comportamiento del resto y modificar los coeficientes estimados. Sin embargo, conviene destacar que los coeficientes a (ver cuadro 5) del procedimiento de Johansen (1988) sugieren que la retroalimentación entre los precios y el tipo de cambio es la de mayor relevancia para el rechazo de la hipótesis de exogeneidad débil. Este resultado es consistente con la presencia de espirales inflacionarias asociadas al ciclo devaluación y alza de precios (Gil-Díaz y Ramos Tercero, 1988) y con la hipótesis de paridad de poder de compra.

EI rechazo de la hipótesis de exogeneidad débil confirma que si bien los efectos por separado de cada una de las variables consideradas en el modelo son relativamente pequeños, ello es consecuencia de que normalmente estas variables se mueven conjuntamente y por tanto, que es el efecto asociado lo que importa. Sin embargo, el uso de series 1(2) y el número de observaciones reducen significativamente el poder de esta prueba. Con el propósito de estudiar con mayor detalle esta situación se estimó el modelo econométrico final para detectar sus propiedades de estabilidad estructural. De este modo, se analizó la hipótesis de exogeneidad débil como una derivación de las propiedades de estabilidad estructural o de superexogeneidad (Ericsson e Irons, 1994).

Cuadro 5: Coeficientes a del procedimiento de Johansen.

\begin{tabular}{ccccc}
\hline & $P_{t}$ & $w_{t}$ & $s_{t}$ & $m_{t}$ \\
\hline$P_{t}$ & -1.0 & 0.5 & 0.3 & 0.2 \\
$W_{t}$ & -1.3 & 0.4 & 0.5 & 0.4 \\
$s_{t}$ & -4.1 & 2.3 & 1.1 & 1.0 \\
$m_{t}$ & 1.2 & -0.7 & -0.3 & -0.3 \\
\hline
\end{tabular}

El vector de cointegración puede utilizarse como mecanismo de corrección de errores con base al teorema de representación de Granger (Engle y Granger, 1987). Esta interpretación corresponde a los modelos econométricos utilizados por Juselius (1992), Franz y Gordon (1993) y De Brouwer y Ericsson (1995). Atendiendo a los resultados de las pruebas de orden de integración de las series, es necesario que las variables incluídas en este modelo sean estacionarias. ${ }^{11}$ Consecuentemente, se em-

\footnotetext{
${ }^{11}$ Esta solución es consistente con las propiedades estadísticas de las series no obstante que algunos otros autores prefieren modelar directamente la tasa de inflación al asumir inicialmente al nivel de
} 
plearon las segundas diferencias de las series de tiempo. El modelo estadístico general (Spanos, 1986) puede definirse ${ }^{12}$ como:

$$
\Delta^{2} \mathrm{p}_{\mathrm{t}}=\sum^{\mathrm{t}-4} \alpha_{\mathrm{t}-\mathrm{i}-1} \Delta^{2} \mathrm{p}_{\mathrm{t}-1}+\sum^{\mathrm{t}=4} \beta_{\mathrm{t}-1} \Delta^{2} \mathrm{~m}_{\mathrm{t}-1}+\sum^{\mathrm{t}=4} \delta_{\mathrm{t}-\mathrm{i}} \Delta^{2} \mathrm{w}_{\mathrm{t}-1}+\sum^{\mathrm{t}=4} \kappa_{\mathrm{t}-\mathrm{i}-1} \Delta^{2} \mathrm{~s}_{\mathrm{t}-\mathrm{i}}+\lambda e c \mathrm{~m}_{\mathrm{t}-4}+\mathrm{u}_{\mathrm{t}}
$$

donde $\mathrm{ecm}_{\mathrm{t}-4}=\mathrm{p}_{\mathrm{t}}-.26 \mathrm{~m}_{\mathrm{t}}-.52 \mathrm{w}_{\mathrm{t}}-.31 \mathrm{~s}_{\mathrm{t}}$

La estimación de la ecuación (24) permite obtener un modelo estadístico satisfactorio ya que los valores proyectados simulan adecuadamente a los valores reales $(\mathrm{R} 2=.95)$, la prueba para autocorrelación del multiplicador de Lagrange con cuatro rezagos $[\mathrm{LM}(4)]$ no rechaza la hipótesis nula $[\mathrm{F}(4,12)=.2 .62]$, la prueba de heteroscedasticidad autoregresiva $(\mathrm{ARCH})$ con cuatro rezagos no rechaza la hipótesis nula $[\mathrm{F}(4,8)=.18] \mathrm{y}$, la prueba de normalidad de Jarque-Bera (JB) no rechaza tampoco la hipótesis nula en la distribución de los errores $\left[\mathrm{X}^{2}(2)=1.35\right]$ (Doornick y Hendry, 1997a, 1997b). De este modo, los residuales no muestran ningún patrón sistemático indicando que toda la información disponible sobre el fenómeno en cuestión está incluída en el modelo general. Así, la metodología de lo general a lo específico permite obtener el siguiente modelo econométrico final:

$$
\begin{aligned}
& \Delta^{2} \mathrm{p}_{\mathrm{t}}=-.56 \Delta^{2} \mathrm{p}_{\mathrm{t}-1}-.18 \Delta^{2} \mathrm{~m}_{\mathrm{t}-1}+.62 \Delta^{2} \mathrm{w}_{\mathrm{t}-1}+.48 \Delta^{2} \mathrm{w}_{\mathrm{t}-2}+.12 \Delta^{2} \mathrm{~s}_{\mathrm{t}-1}+.09 \Delta^{2} \mathrm{~s}_{\mathrm{t}-4}-.87 \mathrm{ecm} \mathrm{m}_{\mathrm{t}-4}
\end{aligned}
$$

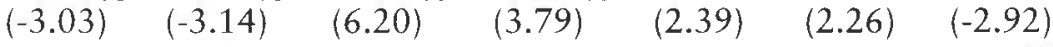

$-.03 \mathrm{Q}_{1}+.02 \mathrm{Q}_{2}+.003 \mathrm{Q}_{3}$

$\begin{array}{lll}(-2.48) & (2.26) \quad(-2.92)\end{array}$

$\mathrm{R}^{2}=.78$

$\mathrm{LM}(4\}: \mathrm{F}(4,26)=.52[.781]$

ARCH: $\mathrm{F}(4,22)=.63[.644]$

]arque-Bera: X2(2) $=1.18(.551]$

White: $\mathrm{F}(17,12)=1.70(.174)$

Prueba de Chow: $\mathrm{F}(4,26)=0.49(.741]$

Prueba de Chow de pronóstico: X2(4) = 4.92(.295]

El modelo econométrico representado en la ecuación (25) es una aproximación adecuada del proceso generador de información (Spanos, 1986). Esto es, todas las variables incluídas son estadísticamente significativas como lo indican las pruebas de t. El coeficiente de decerminación es bastante elevado. El coeficiente del mecanismo de corrección de errares tiene signo negativo y es estadísticamente significativo. Esta es consistente con la teoría económica (Engle y Granger, 1987) e indica que las divergencias de los agentes económicos sobre sus posiciones de equilíbrio se corrigen en el periodo actual mediante modificaciones en la casa de

precios como una serie 1(1) (Brouwer y Ericsson, 1995). Sin embargo, la fuerte variabilidad de la inflación en México hace difícil utilizar esta solución.

12 También Webb (1995), Mehra (1991) y Galindo (1997) utilizan la segunda diferencia de la serie como consecuencia de sus propiedades estadísticas. 
crecimiento de la inflación. Asimismo, los errares del modelo no muestran ningún comportamiento estadístico sistemático según las pruebas de autocorrelación y heteroscedasticidad y, no rechazan la hipótesis de normalidad. Adicionalmente, el modelo muestra una notable estabilidad estructural considerando la fuerte volatilidad de la variable modelada. En efecto, las pruebas de estabilidad estructural de Chow y de Chow de pronóstico no rechazan la hipótesis nula. Además, las estimaciones recursivas del modelo expresan una alta estabilidad estructural con la excepción de 1995 como se observa en las gráficas 1, 2 y 3.

Gráfica 1: Residuales obtenidos por estimación recursiva

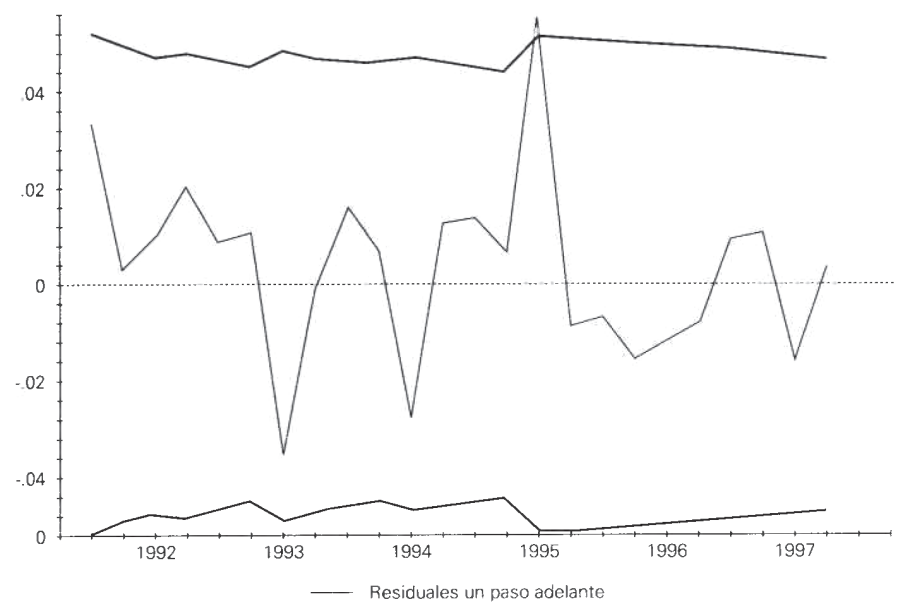

Gráfica 2: Prueba de Chow de un paso adelante

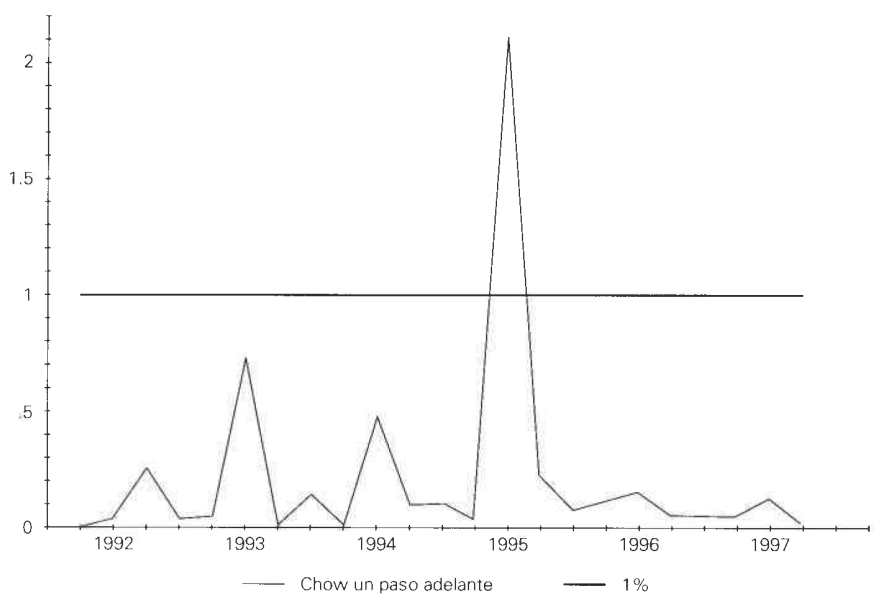




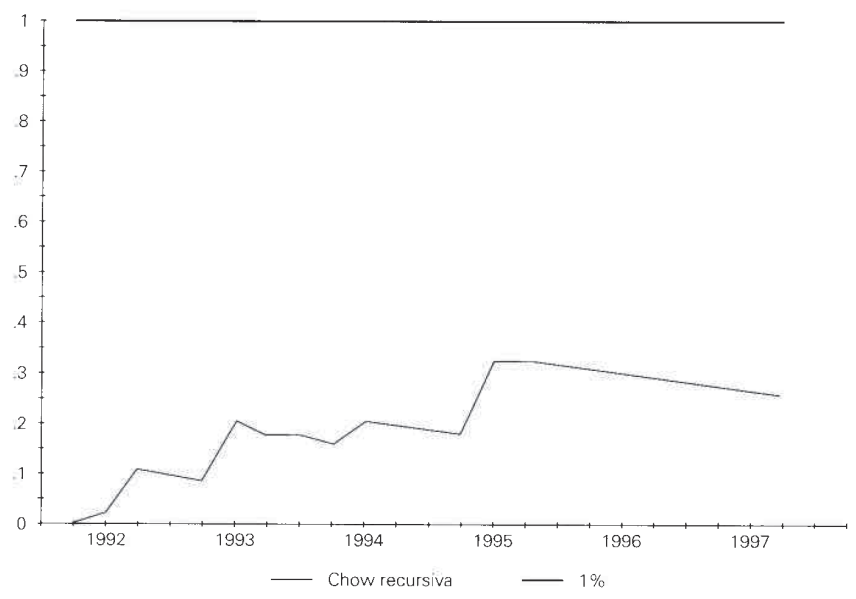

Las propiedades estadísticas del modelo anotadas anteriormente se reflejan en la alta capacidad del modelo para simular el comportamiento histórico de los datos, en la adecuada capacidad del modelo para la realización de pronósticos en los períodos simulados y, en que no existe una diferencia estadísticamente significativa entre los valores reales y los proyectados (cuadro 6).

Gráfica 4: Valores reales y proyectados

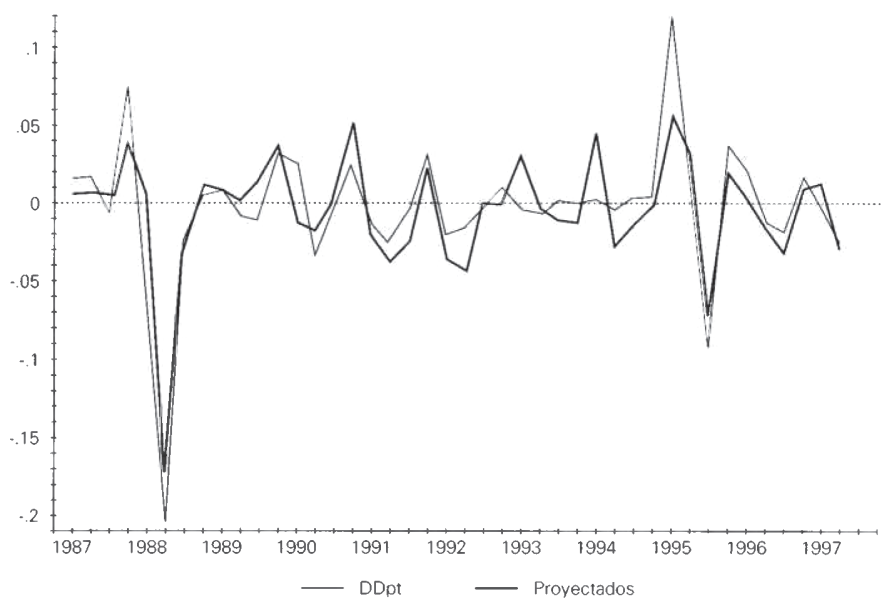


Cuadro 6: Análisis de pronósticos de un período adelante .

\begin{tabular}{lccccc}
\hline Período & Real & Proyectado & $D^{2} p-D^{2} p^{e}$ & S.E proyectado & valor t \\
\hline $1996(1)$ & .0032 & .0566 & -.0534 & .0405 & -1.31 \\
$1996(2)$ & -.0179 & -.0333 & .0154 & .0416 & 0.36 \\
$1996(3)$ & -.0190 & -.0303 & .0112 & .0340 & 0.33 \\
$1996(4)$ & .0157 & .0133 & .0022 & .0326 & 0.06 \\
\hline
\end{tabular}

\section{CONCLUSIONES Y CONSIDERAC IONES GENERALES}

EI nivel de precios tiene una relación estable de largo plazo con el salario mínimo nominal, el agregado monetario $\mathrm{m} 2$ y el tipo de cambio nominal. Los coeficientes obtenidos permiten identificar las ponderaciones directas de los movimientos de estas variables sobre el índice de precios y permiten obtener una visión más realista de las formas de ajuste simultáneo entre las variables consideradas. Cabe destacar que este modelo incorpora las presiones inflacionarias generadas en los mercados monetario, laboral y cambiaria suponiendo que el efecto del ingreso está mediado por el efecto riqueza del agregado monetario.

La prueba de exogeneidad débil indica que existe una retroalimentación entre estas variables que debe tomarse en consideración. Esta es, la formación estadística de cada una de estas series no es independiente y por tanto, deben considerarse los movimientos conjuntos de las mismas para no perder información relevante al proceso de formación de precios. Además, esta retroalimentación permite explicar que el efecto de cada una de las variables por separado sea relativamente menor al esperado en los modelos parciales, en donde podría asumirse coeficientes cercanos a uno. Esta es, en análisis parciales se espera un ajuste de uno a uno entre el agregado monetario y los precios, el salario mínimo nominal y los precios y, el tipo de cambio y los precios. Sin embargo, el modelo muestra que por el movimiento conjunto de todas estas variables los efectos directos o ponderados de cada coeficiente son menores a uno aunque el total sea mayor a la unidad. El movimiento conjunto de estas variables es más realista considerando por ejemplo que una devaluación del tipo de cambio o un aumento en los castos laborales unitarios está normalmente acompanado por un aumento en los agregados monetarios.

Con base en el teorema de representación de Granger puede obtenerse un modelo econométrico especificado para la doble diferencia del nível de precios que aproxima adecuadamente al proceso generador de información (Spanos, 1986). Destaca que este modelo se construyó utilizando la segunda diferencia del índice de precios según las propiedades estadísticas de la serie de tiempo. En México, la inestabilidad de la rasa de inflación se traduce en cambias estructurales dificultando su modelación. Sin embargo, el uso de la serie en segundas diferencias produce 
un comportamiento más sistemático en la media y la varianza que facilita la construcción de un modelo econométrico con estabilidad estructural en sus parámetros.

Finalmente, la existencia de una relación de equilíbrio entre el nivel de precios, el salario mínimo nominal, el tipo de cambio y un agregado monetario puede utilizarse para validar el uso de anelas nominales. Precisamente el nivel de precios de equilíbrio consistente con las otras variables representa una guía sobre la trayectoria futura del nivel de precios y, por tanto, el banco central está en condiciones de monitorear la evolución de las otras variables y conocer si existen condiciones para una alza de precios y las condiciones monetarias que la validen. Sin embargo, es conveniente considerar que la complejidad del ajuste dinámico y la retroalimentación entre las variablcs cstudiadas hacen difícil realizar ajustes exactos sobre el comportamiento de los precios en el futuro.

\section{REFERENCIAS}

ACEITUNO, C. e I. RUPRAH (1982), “Déficit público e inflación” en Economía Mexicana, n. 4. pp.47-60. ARELLANO, R. y E. GONZÁLEZ (1993), "Dinámica de la inflación: un análisis econométrico del ajuste heterodoxo mexicano" en Estudios Económicos, vol. 8, n. 2. pp. 249-261.

AROCHE, F. (1993), "Endogenous prices for input-output models: a note” en Economic Systems Research, vol. 5, n. 4.

BARRO, R.J. (1979), “Money and output in Mexico, Colombia and Brazil”, en J. Behrman y J. Hanson (eds.), Short term macroeconomic policy in Latin America, Cambridge, Massachussets, pp. 177 200.

BOSCHEN, S. y K. E. TALBOT (1991), "Monetary base growth, deposit growth and inflation in the postwar United States”, Journal of Business, 64, pp. 313-338.

BRUNO, M. (1979), "Price and output adjustment”, Journal of Monetary Economics, vol. 5, n. 2, pp. 187-211.

CARLIN, Wendy y David SOSKICE (1990), Macroeconomics and the wage bargain: a modern approach to employment, inflation and exchange rate, Oxford University, New York, p. 482.

CASAR, J., M. DEHESA,J. ROSS y ALEJANDROVAZQUEZ (1979). "La hipotesis de precios normales y su aplicacion al sector manufacturero", Economia Mexicana, Centro de Investigación y Docencia Económica, n. 1, pp. 121-146.

CORBO, V. (1985), "International prices, wages and inflation in an open economy: a Chilean model”, Review of Economics and Statistics, vol. 67, n. 4, pp. 564-573.

CUTHBERTSON, K., S. HALL y M. TAYLOR (1992), Applied econometric techniques, Philip Allan.

DAVILA, J., A. IZE y J. MO RALES (1984), “Fuentes del proceso inflacionario en México: análisis de causalidad” en A. Ize y G. Vera (comp.), La inflactión en México, El Colegio de México, pp. 57-70.

DE BROUWER, G. y N. R. ERICSSON (1995), "Modelling inflation in Autralia”, Board of Governor of the Federal Reserve System, International Finance, Discussion Papers, n. 530, noviembre.

DÍAZ ALEJANDRO, C. F. (1965), Exchange rate devaluation in a semi-industrial country: the experience of Argentina 1955-1961, Cambridge University Press., pp. 115-125.

DICKEY, D. y W.A. FULLER (1981), "Likelihood ratio statistics for autoregressive tiem series with unit root”, Econometrica, vol. 49, n. 4, pp. 1.057-1.072.

DOORNICK, J. A. y D. F. HENDRY (1997a), PcFiml 9.0, International Thompson Business Press.

DOORNICK, J. A. y D. F. HENDRY (19976), PcGive 9.0, Internacional Thompson Business Press.

ENGLE, R. F. (1983), "Wald, likelihood ratio, and lagrange multiplier tests in econometrics" en Handbook of Econometrics, Z. Griliches y M. D. Intriligator (eds.), vol. II. North-Holland Puhlishing Co. Capítulo 13, pp. 775-826. 
ENGLE, R. F. y C. W. J. GRANGER (1987), “Co-integration and error correction: representation, estimation and testing”, Econometrica, vol. 55, n. 2, pp. 251-276.

ENGLE, R. F., D. F. HENDRY y J. F. RICHARD (1983), “Exogeneity”, Econometrica, vol. 51, n. 2, pp. 277-304.

ERICSSON N. R. y J. S. IRONS (1994), Testing exogeneity, Oxford University Press.

FAIRLIE, A. (1992), "Inflación y ajuste estructural en América Latina” en Investigación Económica, vol. LI, n. 202, pp. 79-108.

FRANZ, W. y R.J. GORDON (1993), “German and American wage and price dynamics: differences and common themes", European Economic Review, 37(4), pp. 719-762.

GALINDO, L. M. (1997), "El modelo P* como indicador de la política monetaria en una economías con alta inflación”, El Trimestre Económico, vol. LXIV, n. 2, abril-junio, pp. 221-239.

GIL-DÍAZ, F. y R. RAMOS TERCERO (1988), “Lecciones desde México”, en M. Bruno, G. Di Tella, R. Dornbusch y S. Fiscer (comp.), Inflación y estabilización: la experiencia de Israel, Argentina, Brasil, Bolivia y México, Lecturas 62, Fondo de Cultura Económica, pp. 425-457.

GÓMEZ OLIVER, A. (1978), Dinero, Inflación y comercio exterior en México, CEMLA.

GRANGER, C. W. J. (editor), (1990), Modelling economic series: readings in econometric methodology, Oxford University Press.

HALL, S. G. y A. MILNE (1994), “The relevance of P-Start analysis to UK monetary policy”, Economic Journal, n. 104, May, pp. 597-604.

HALLMAN, J. J., R. D. PORTER y D. H. SMALL (1991), "Is the price level tied to the M2 monetary aggregate in the long run?", American Economic Review, vol. 81, n. 4, pp. 841-858.

HARBERGER, A. C. (1963), "The dynamics of inflation in Chile", en Measurement in Economics: studies in mathematical economics and econometrics in memory of Yehuda Grunfeld, Stanford, pp. 219- 230.

HATANAKA, M. (1996), Time series-based Econometrics, Unit Roots and Co-integration, Oxford University Press.

IZE, A. y G. VERA (1984), La inflación en México, Introducción, El Colegio de México.

IZE, A. y J. SALAS (1984), "El comportamiento macroeconómico de la economia mexicana entre 1961 y 1981: especificaciones alternativas y pruebas de hipótesis” en A. Ize y G. Vera (comp.), La inflación en México, El Colegio de México, pp. 171-231.

JIMENEZ, F. y C. ROCES (1979), "Precios y márgenes de ganancia en la industria manufacturera mexicana”, Economia Mexicana, Centro de Investigacion Economica, n. 3, pp. 183-252.

JOHANSEN, S. (1988), "Statistical analysis of co-integrating vectors", Journal of Economics Dynamics and Control, n. 12, pp. 231-254.

JOHANSEN, S. (1992), "An 1(2) co-integration analysis of the purchasing power parity between Australian and the United States" en C.P. Hargreaaves (ed.) Macroeconomic modelling in the long run, capítulo 9, Edward Elgar, pp. 229-249.

JUSELIUS, K. (1992), "Domestic and foreign effects on prices in an open economy: the case of Denmark", Journal of Policy Modelling, vol. 14, n. 4, pp. 401-428.

LAYARD, R., S. NICKELL y R. JACKMAN (1996), La crisis del paro, Alianza editorial.

LEIDERMAN, L., N. LIVIATAN y A. THORNE (1995), "Shifting nominal anchors: the experience of Mexico" en Economía Mexicana, vol. IV, n. 2. pp. 197-238.

LEVY, S. (1984), “Un modelo de simulación de precios para la economía mexicana”, en A. Ize y G. Vera (comp.), La inflación en México, El Colegio de México, pp. 249-277.

LINDBECK, A. (1979), "Imported and structural inflation and aggregate demand: the Scandinavian model reconstructed", en A. Lindbeck (ed.), Inflation and Employment in open economies, Amsterdam, North Holland Publishing, capítulo 1, pp. 13-40.

LIZONDO, S. (1992), “The dynamics of inflation, 1988-1991 “, en C. Losar y E. Kalter (ed.), Mexico: the strategy to achieve sustained economic growth, Fondo Monetario Internacional, documentos ocasionales, n. 99, pp. 73-87.

MARTNER, R. y D. TITELMAN (1990), “Inflación y nivel de actividad en Chile: una aplicación del modelo de corrección de errores”, El Trimestre Económico, vol. LVII, n. 225, pp. 195-239. 
MARTNER, R., T. TITELMAN y A. UTHOFF (1995), “Componentes internos y externos de la inflación en Chile: un enfoque de cointegración”, en F. G. Morade y F. Rosende (ed.) Análisis empírico de la inflación en Chile, Instituto de Economía Pontifícia Universidad Católica de Chile y Programa de Posgrado en Economia ILADES/Georgetown University.

MEHRA, Y. P. (1991), "Wage growth and the inflation process: an empirical note", American Economic Review, vol. 81, n. 4, septiembre, pp. 931-937.

NOYOLA, J. (1987), "El desarrollo económico y la inflación en México y otros países latinoamericanos” en Materiales Investigación Económica, Facultad de Economía, UNAM, pp. 67-107.

PHILLIPS, P.C.B. y P. PERRON (1988), “Testing for unit root intime series regression”, Biometrica, vol. 75, pp. 335-346.

PINTO, A. (1968), "Raíces estructurales de la inflación en América latina”, El Trimestre Económico, vol. XXXV, n. 137, pp 63-74.

PREBISCH, R. (1982), "El falso dilema entre desarrollo económico y estabilidad monetaria”, en A. Gurrieri (de.), La obra de Prebisch en la CEPAL, Lecturas 46, Fondo de Cultura Económica, pp 9-71.

QUAH, D. y S. D. VAHEY (1995), “Measuring core inflation”, Economic Journal, vol. 105, septiembre, pp. 1130-1144.

ROSS, J. (1981), “La inflación: la experiencia de la década de los setenta”, en R. Cordera (ed.), Desarrollo y crisis de la economía mexicana, Lecturas 39, pp. 422-438.

ROSS, J. (1984), “El proceso inflacionario en México”, en A. Ize y G. Vera (comp.), La inflación en México, El Colegio de México, pp. 131-145.

RUPRAH, I. (1981), “Notas sobre la relación entre dinero e inflación” en Economía Mexicana, n. 3. pp. $165-182$.

RUPRAH, I. (1982), "El teorema de la paridad del poder adquisitivo: inflación y tipo de cambio" en Economía Mexicana, n. 4, pp. 61-76.

RUPRAH, I. (1984 , “Es la inflación, siempre y en todos lados un fenómeno monetario?”, en A. Izey y G. Vera (comp.), La inflación en México, EL Colegio de México, pp. 41-56.

SACHS, J y F. LARRAIN (1993), Macroeconomics in the global economy, Printece Hall Inc.

SAHLING, L. (1977), "Price behavior in U.S. manufacturing: an empírical analysis of the spedd of adjustment”, American Economic Review, vol.67, n. 5, pp. 911-925.

SALAS, J. y A. IZE (1984), "Dinero, precios y producto: un analisis de autorregresión vectorial para México”, en A. Ize y G. Vera (comp.), La inflación en México, El Colegio de México, pp. 71-86.

SAWYER, M., S. AARONOVITCH y P. SAMSON (1982), "The influence of cost and demand changes on the rate of change of prices", Applied Economics, 14, pp. 195-209.

SPANOS, A. (1986), Statistical foundations of econometric modelling, Cambridge University Press.

TURNOVSKY, S. J. (1977), Macroeconomic analysis and stabilization policy, Cambridge University Press.

VOGEL, R. (1974), “The dynamics of inflation in Latin America, 1950-19 69”, American Economic Review, vol. LXIV, n. 1, pp. 102-114.

WEBB, R. H. (1995), "Forecasts of inflation from VAR models”, Journal of Forecasting, vol. 14, pp. 267-285.

WICKENS, M. (1993), "Interpreting co-integrating vectors and common stochastic trends ", en Documento de discusión núm. 14, Business School, Centre for economic forecasting, London.

YACAMAN, J. M. (1984), “Análisis de la inflación en México”, en A. Ize y G. Vera (comp.), La inflación en México, El Colegio de México, pp. 147-158. 


\section{APENDICE}

Cuadro 1.a : Valores característicos

0.66535

0.37582

0.12374

Cuadro 2.a: Pruebas estadísticas del VAR

\begin{tabular}{ccccc}
\hline & $p_{t}$ & $W_{t}$ & $m_{t}$ & $s_{t}$ \\
\hline $\begin{array}{c}\text { Autocorrelación: } \\
\text { LM(4) }\end{array}$ & $F(4,22)=.14$ & $F(4,22)=2.45$ & $F(4,22)=1.66$ & $F(4,22)=.73$ \\
$\begin{array}{c}\text { Heteroscedastici- } \\
\text { dad: ARCH(4 }\end{array}$ & $F(3,20)=.97$ & $F(3,20)=.68$ & $F(3,20)=.35$ & $F(3,20)=.03$ \\
$\begin{array}{c}\text { Normalidad: } \\
\text { Jarque-Bera }\end{array}$ & $X^{2}(2)=1.19$ & $X^{2}(2)=2.11$ & $X^{2}(2)=6.28^{*}$ & $X^{2}(2)=27.11^{* *}$ \\
\hline
\end{tabular}

\section{ESPECIFICACIÓN DE LAS SERIES}

\begin{tabular}{|c|c|c|c|c|}
\hline Clave & Descripción & $\begin{array}{l}\text { Periodicidad } \\
\text { original }\end{array}$ & Fuente & Unidad \\
\hline$M$ & $\begin{array}{l}\text { Agregado monetario total M2 } \\
\text { (último mes del trimestre) }\end{array}$ & Mensual & $\begin{array}{l}\text { Indicadores Económicos } \\
\text { Banco de México }\end{array}$ & $\begin{array}{l}\text { Millones } \\
\text { de pesos }\end{array}$ \\
\hline$P$ & $\begin{array}{l}\text { Indíce de precios al consumidor } \\
\text { (último mes del trimestre) }\end{array}$ & Mensual & $\begin{array}{l}\text { Indicadores Económicos } \\
\text { Banco de México }\end{array}$ & Base 1994 \\
\hline$S$ & $\begin{array}{l}\text { Tipo de cambio interbancario } \\
\text { (último mes del trimestre) }\end{array}$ & Diario & $\begin{array}{l}\text { Indicadores Económicos } \\
\text { Banco de México }\end{array}$ & $\begin{array}{l}\text { pesos por } \\
\text { dólar }\end{array}$ \\
\hline W & $\begin{array}{l}\text { Salario mínimo diario general } \\
\text { (último mes del trimestre) }\end{array}$ & Mensual & $\begin{array}{l}\text { Comisión Nacional de } \\
\text { Salarios Mínimos }\end{array}$ & pesos \\
\hline
\end{tabular}

